\title{
Ethinylestradiol/Chlormadinone Acetate for Use in Dermatological Disorders
}

\author{
Mercedes Gómez Vázquez, ${ }_{1}^{1}$ Ramón Navarra Amayuelas, ${ }_{1}^{1}$ Marta Lamarca, ${ }^{2}$ Laura Baquedano, ${ }^{2}$ Sebastián Romero Ruiz, \\ Eduardo Vilar-Checa ${ }^{4}$ and Maria D. Iniesta ${ }^{5}$ \\ 1 Deparment of Dermatology, Hospital Municipal of Badalona, Badalona, Barcelona, Spain \\ 2 Department of Gynecology, Miguel Servet University Hospital, Zaragoza, Spain \\ 3 Hospital of Móstoles, Department of Obstetrics and Gynecology Móstoles, Madrid, Spain \\ 4 University Hospital of Elche, Elche, Alicante, Spain \\ 5 Division of Molecular Medicine and Genetics, Department of Internal Medicine, University of Michigan, Michigan, USA
}

\section{Abstract}

\section{Introduction}

Female alopecia, hirsutism, acne and polycystic ovary syndrome (PCOS) share a common thread: the endocrine abnormality of hyperandrogenism. Hirsutism and acne, and to a lesser extent, female alopecia, are all clinical signs of hyperandrogenization. ${ }^{[1]}$ PCOS is considered primarily to be a syndrome of hyperandrogenism. ${ }^{[1]}$ The majority of patients with hirsutism or unwanted hair growth (75-80\%) have PCOS, and approximately $20-40 \%$ of patients with persistent acne only have PCOS; ${ }^{[1]}$ approximately $10 \%$ of women with alopecia also only have PCOS. ${ }^{[1]}$

\begin{abstract}
The diagnosis and management of four cases of dermatological disorders, most of which are related to the endocrine disorder of androgen excess, are presented. Combined oral contraceptives (COCs) may be useful when well-tolerated hormonal therapy and/or when contraception is required. A female patient with androgenetic alopecia or female pattern balding, without underlying hyperandrogenism, was treated with ethinylestradiol/chlormadinone acetate (EE/CMA) $0.03 \mathrm{mg} / 2 \mathrm{mg}$ for 6 months and experienced stabilization of hair loss (case report 1). A patient who had previously received a COC for an irregular menstrual pattern but again experienced irregular menses and also acne after stopping treatment was diagnosed with acne associated with polycystic ovary syndrome (PCOS) [case report 2]. After 6 month's treatment with EE/CMA $0.03 \mathrm{mg} / 2 \mathrm{mg}$, this patient had fewer acne lesions and became eumenorrheic. A third patient who had excess hair since childhood was diagnosed with idiopathic hirsutism (no underlying gynecological or endocrinological disorder was found) and was treated with EE/CMA $0.03 \mathrm{mg} / 2 \mathrm{mg}$ (case report 3). Less hair growth was reported after 6 months' treatment. Case report 4 describes a patient who presented with oligomenorrhea and acne. She was diagnosed with PCOS with acne, seborrhea and mild hirsutism. Treatment with $\mathrm{EE} / \mathrm{CMA} 0.03 \mathrm{mg} / 2 \mathrm{mg}$ for 6 months resulted in improvements in her facial acne, seborrhea and hirsutism; she also became eumenorrheic. These four cases illustrate that EE/CMA may be a useful and well tolerated treatment option in the management of patients with dermatological disorders with or without hyperandrogenization.
\end{abstract}


laboratory testing, their condition improves after treatment with oral anti-androgens, confirming at least some involvement of androgens in the pathogenesis of this kind of alopecia. ${ }^{[6,7]}$

Hirsutism in women is excessive terminal hair appearing in a male (androgen-dependent) sexual pattern, ${ }^{[8]}$ and is associated with psychological distress. ${ }^{[9]}$ Approximately half of the cases of hirsutism in women are due to hyperandrogenism. ${ }^{[8]}$ When hirsutism occurs in the absence of excess androgen it is termed 'idiopathic hirsutism' ${ }^{[8]}$ Hirsutism should be distinguished from hypertrichosis, which is an increase in body hair beyond the normal variation of the patient's reference group, may be congenital or acquired, and is associated with abnormal hair growth that is either localized or generalized. ${ }^{[10]}$

Another clear clinical signal of hyperandrogenism is acne. ${ }^{[1]}$ Acne lesions (papules, pustules, nodules) form through the interaction of excess sebum production, sebaceous follicle obstruction and bacterium Propionibacterium acnes proliferation and subsequent inflammation. ${ }^{[12]}$ There is a correlation between excess sebum production and androgen levels, but the sensitivity of the sebaceous glands to hormonal stimulation also contributes to the formation of acne, ${ }^{[9]}$ as increased sensitivity results in the overproduction of sebum.

PCOS is one of the most common endocrine abnormalities of women of reproductive age, affecting 6-8\% of women worldwide. ${ }^{[1]}$ PCOS is thought to be associated with an increased risk of infertility, endometrial carcinoma, obesity, type II diabetes mellitus, dyslipidemia, hypertension and possible cardiovascular disease. ${ }^{[1]}$ Characteristic features of PCOS are menstrual disorders (oligo or anovulation), clinical and/or biochemical signs of hyperandrogenism and polycystic ovaries. ${ }^{[1]}$ Not all patients with PCOS have elevated androgen levels, thus serum measurements of androgen levels are never the sole criterion for diagnosis. ${ }^{[1]}$

There is rationale for the use of a treatment that targets the underlying hyperandrogenism of acne, hirsutism and FPB in patients with or without PCOS. The four cases presented in this series highlight the potential use of the monophasic combined oral contraceptive ethinylestradiol/chlormadinone acetate (EE/CMA) [Balianca, Belara], in women who would also like birth control. EE/CMA is approved in Europe for contraception, ${ }^{[13]}$ therefore discussion of its use in these cases is off-label.

\section{Case Report 1}

Mercedes Gómez Vázquez, Ramón Navarra Amayuelas

A 32-year-old woman, with no other history of clinical significance, developed slow and progressive hair loss over a period of 6 months before her visit. The patient reported a considerable decrease in hair volume, with no other associated symptoms except the negative influence of this hair loss on her self-image and mental state. There was no family history of hair loss and no history of drug use. Possible factors that may precipitate or exacerbate alopecia, including chronic illness, nutritional alteration, metabolic and endocrinolic alteration, recent surgical intervention and medical treatment, were excluded.

Clinical examination showed hair loss in the median line of the head, with a Christmas tree pattern of hair distribution, Olsen scale type II, with greater involvement of the central line and conservation of the frontal hair implantation line (figure 1). Pull test was negative. No signs of excess sebum production were observed. The patient had no present terminal hair loss from other locations and no nail changes. Effluvium was ruled out by the absence of a diffuse loss of scalp hair, the absence of laboratory abnormalities, and no history of drug use. Laboratory testing included a hemogram (complete blood count), assessment of iron and ferritin levels, and of the following hormones: thyrotropin hormone, thyroid T4, follicle-stimulating hormone (FSH), luteinizing hormone ( $\mathrm{LH}$ ), prolactin, estrogen, free testosterone and dehydroepiandrosterone sulfate (DHEAS). All test results were within the

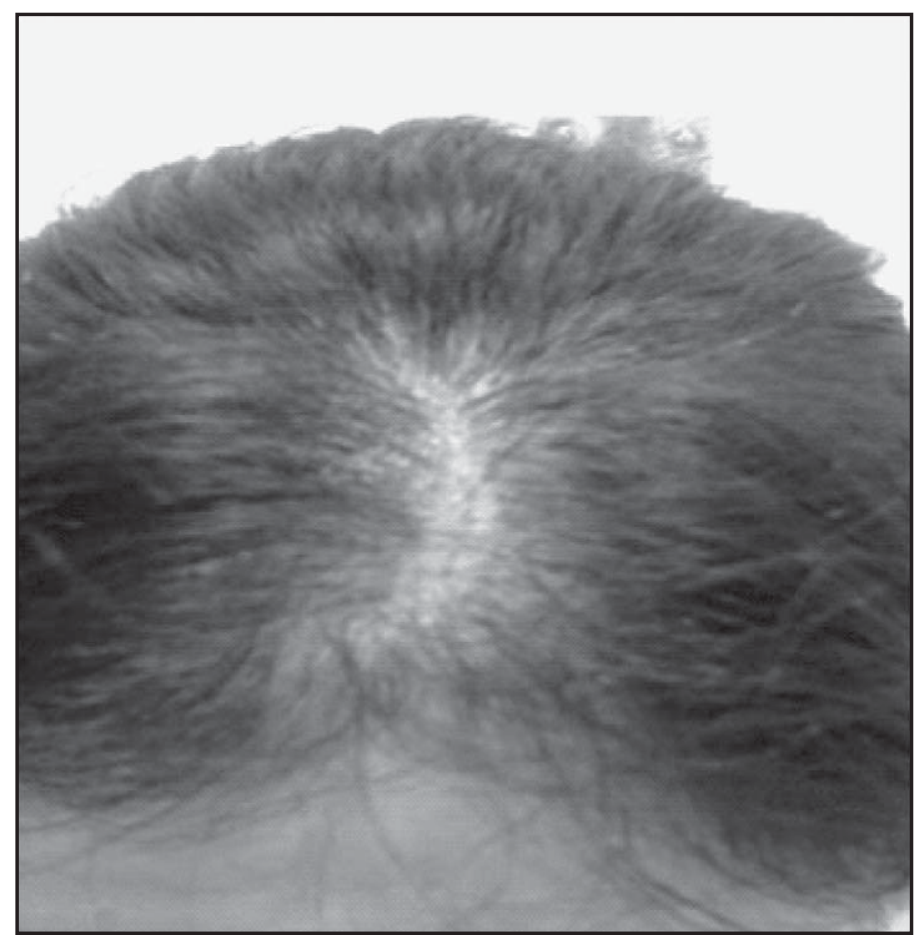

Fig. 1. Frontovertical alopecia or hair loss with a triangular or Christmas tree pattern. 


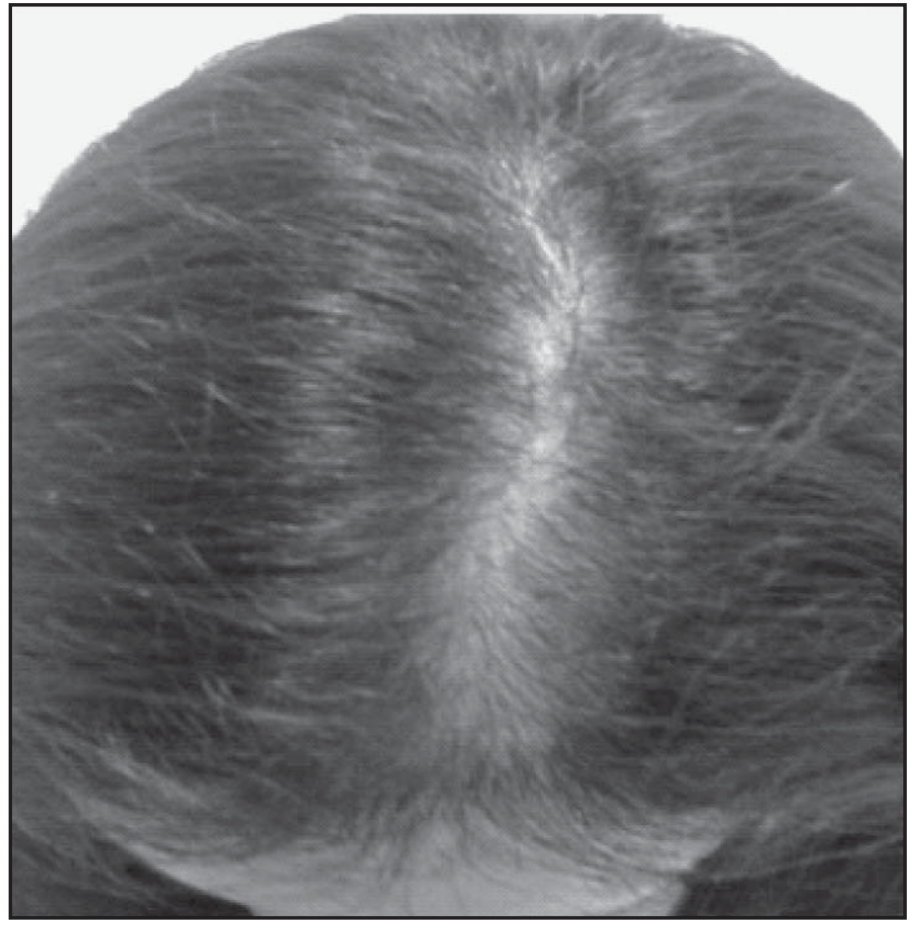

Fig. 2. Hair regrowth after 1 year of treatment showing slight improvement with partial recovery of central line.

normal range. Gynecological examinations showed no gynecological conditions.

The patient was diagnosed with FPB with no underlying endocrine disorder. Treatment with oral EE/CMA $0.03 \mathrm{mg} /$ $2 \mathrm{mg}$ plus daily application of topical minoxidil $2 \%$ in a $2 \mathrm{~mL}$ aqueous solution was initiated. After 3 months, minoxidil treatment was suspended because of local adverse drug reaction, erythema and irritation. After 6 months' treatment with $\mathrm{EE} / \mathrm{CMA}$, the patient reported subjective improvement in hair loss and mental state. Examination showed stabilization of the hair loss process (Olsen type II). The pull test remained negative. Figure 2 shows hair regrowth after 1 year of treatment (Olsen type I).

\section{Case Report 2}

\section{Marta Lamarca, Laura Baquedano}

The female patient was 29 years old with a history of chronic ferropenic anemia receiving oral ferrotherapy, but with no other significant medical or surgical history. Since menarche at the age of 13 years the patient had had an irregular menstrual pattern (3-4/45-79) for which she received ethinylestradiol/ cyproterone acetate $35 \mu \mathrm{g} / 2 \mathrm{mg}$ treatment, which resulted in eumenorrhea. At age 28 years contraceptive treatment was voluntarily stopped. After 1 year without contraceptive treat- ment her menstrual irregularity returned and the patient reported an increase in dermatological lesions.

Physical examination revealed facial acne with over 20 inflammatory lesions and over 30 non-inflammatory lesions (blackheads in the frontal, nasal and chin area). There was no presence of hirsutism. Bodyweight had increased by $6 \mathrm{~kg}$ since the last check-up 1 year previously when the patient was aged 28 years, i.e. when oral contraceptive treatment was voluntarily stopped. Gynecological examination showed that the genitalia, vagina and uterine cervix were of normal appearance. Bimanual examination revealed the uterus in anteflexion was of normal size and morphology, and no adnexal masses were felt. Transvaginal ultrasonography revealed that the uterus in anteflexion measured $73 \times 45 \times 48 \mathrm{~mm}$, the endometrium was $8 \mathrm{~mm}$ and the myometrium was homogeneous. Both ovaries had over 12 follicles each (each follicle $2-9 \mathrm{~mm}$ in size). There was no fluid in the pouch of Douglas. Hormone serum levels were within the normal range (17 beta estradiol $49 \mathrm{pg} / \mathrm{mL}$, progesterone $1.1 \mathrm{ng} / \mathrm{mL}, \mathrm{LH} 18.27 \mathrm{mIU} / \mathrm{mL}$, FSH $6.61 \mathrm{mIU} / \mathrm{mL}$ ) except for androstenedione $(7.3 \mathrm{ng} / \mathrm{mL}$; normal range $0.30-3.30 \mathrm{ng} / \mathrm{mL}$ ). The patient had had irregular menstruation since discontinuing oral contraceptive treatment, and she had not been menstruating for 3 months at the time of the laboratory tests.

The patient was diagnosed with acne associated with PCOS. Treatment with EE/CMA $30 \mu \mathrm{g} / 2 \mathrm{mg}$ was prescribed. After 6 months, her acne improved (less than five inflammatory lesions; less than 15 non-inflammatory lesions).The patient was eumenorrheic (menstrual pattern 2-3/28) and her bodyweight stabilized, $77.4 \mathrm{~kg}$ before treatment and 75.1 at last measurement.

\section{Case Report 3}

\section{Sebastián Romero Ruiz}

A 19-year-old female patient reported excess hair since childhood, which had increased since puberty, but no rapid increase before the visit was reported. The patient was nulliparous, experienced menarche aged 13 years and did not report menstrual disorders (menstrual pattern 4/28-35). The patient's mother had excess hair, treated with laser hair removal, and had experienced two pregnancies and normal childbirths. The patient had no other significant personal or family medical history.

The patient's excess hair was assessed using the FerrimanGallwey scoring system, with a total score of 16 (maximum 36), distributed as follows: upper lip 3, chin 3, thorax (periareolar) 1, abdomen 2, pubis 2 , arms 2 , legs 1 , upper back 1 , lower back 1 . No acanthosis nigricans was found. Her body mass index was 23. Manual gynecological examination results were normal, 
with no hypertrophy of the clitoris or inguinal mass. The gynecological ultrasound revealed a normal genital system, with no suggestion of polycystic ovaries. Laboratory, hemogram and coagulation test results were within the normal range. The following serum hormone levels were assessed in the initial cycle and were all normal: prolactin, thyroid hormones, FSH, LH (LH:FSH ratio of 1.5), estradiol, testosterone, androstenedione, dehydroepiandrosterone, DHEAS, 17.OH. progesterone. Free urine cortisol levels were also normal.

We diagnosed the patient with idiopathic hirsutism. Treatment was initiated with oral EE/CMA $0.03 \mathrm{mg} / 2 \mathrm{mg}$. After 6 months' treatment, the Ferriman-Gallwey score decreased to 11 (upper lip: 2, chin 1, thorax, 1 abdomen 1, pubis 2, arms 2, legs 1 , upper back 0 , lower back 1 ), with clear improvements in the facial area.

\section{Case Report 4}

\section{Eduardo Vilar-Checa, Maria D. Iniesta}

A 20-year-old woman presented in July 2010 with oligomenorrhea (menstrual pattern 3/35-50) and progressive acne since menarche (13 years). The patient was not taking any medications. The patient's menstrual pattern was $3 / 35-50$ and her longest period of amenorrhea had been 4 months. There was no history of an abnormal pap smear and the last one was obtained in July 2010. The patient denied pain with menstruation or intermenstrual bleeding. The patient also sought counselling for an appropriate contraceptive method. The patient and her parents' medical history were otherwise unremarkable.

Dermatological examination revealed the presence of acne, especially on her face and only to a slightly mild degree on her back; the lesions were mainly papulopustular lesions, particularly on her face. She also showed slight hirsutism in the form of dark terminal hairs involving the chin. A significant increase of the seborrheic secretion on the face was also noticed upon clinical assessment.

There were no other signs of virilization. Gynecological examination revealed that the vulva, urethra, vagina and cervix were normal. On bimanual examination, no nodularity, mass or tenderness was noted on the uterus or ovaries. The uterus was normal in size, shape and mobility and no adnexal masses were noted. The cul-de-sac was smooth. Breasts were without dominant masses, skin changes or nipple discharge. Physical examination suggested an otherwise healthy individual (body mass index 22.86).

Gynecological ultrasound revealed a normal-sized uterus in anteversion. Both ovaries had multiple follicles (14 follicles of $4-6 \mathrm{~mm}$ in diameter in the right ovary and 16 of the same di- mensions in the left ovary). The volume of both ovaries was over $10 \mathrm{~mL}$ and the ovarian stroma was hyperechogenic. A complete blood count, coagulation testing and laboratory testing (including glucose and cholesterol levels) showed normal ranges, including a euglycemic-normoinsulinemic profile. Baseline hormone levels on the third day of the menstrual cycle were as follows: FSH $3.25 \mathrm{mUI} / \mathrm{mL}$, LH $10.90 \mathrm{mUI} / \mathrm{mL}$ (with a ratio LH:FSH >3), estradiol $60 \mathrm{pg} / \mathrm{mL}$, prolactin $15.10 \mathrm{ng} / \mathrm{mL}$ (normal values $4.50-40$ ), total testosterone $0.59 \mathrm{ng} / \mathrm{mL}$ (normal values $0.20-0.90$ ), DHEAS $270 \mu \mathrm{g} / \mathrm{dL}$ (normal values $10-340$ ) and androstenedione $6.02 \mathrm{ng} / \mathrm{mL}$ (normal 0.30-3.3).

The patient was diagnosed with PCOS. Treatment with EE/CMA $0.03 \mathrm{mg} / 2 \mathrm{mg}$ was initiated. After 6 months' treatment, there were slight improvements in acne with the number of acne lesions decreased almost to half on her face, and a marked decrease in seborrheic secretion and facial hirsutism. The patient denied any laser hair removal or waxing of her face subsequent to treatment initiation. Androstenedione levels decreased from $6.02 \mathrm{ng} / \mathrm{mL}$ to $3.33 \mathrm{ng} / \mathrm{mL}$. The patient's menstrual cycle control improved, and cycles normalized without spotting at any time (menstrual pattern 3/28). At 10 months after treatment initiation, the patient remains on treatment with EE/CMA.

\section{Conclusions}

Differential diagnoses were ruled out in each case reported. The patient in case 1 had FPB in the most commonly reported pattern of hair loss in women: the Christmas tree pattern, first described by Olsen, in which there is intensive hair loss in the median line of the head that progressively increases towards the frontal area. ${ }^{[7]}$ Diffuse telogen effluvium (the patient had normal iron/ferritin and thyroid hormone levels) ${ }^{[5]}$ alopecia areata (absence of diffuse or patches of hair loss on the scalp, absence of terminal hair loss in other locations, abscense of nail changes [pitting] and a negative pull test), underlying hyperandrogenism (no clinical signs of excessive body hair growth, seborrhea, acne, ${ }^{[4]}$ and testosterone levels were normal) and PCOS (no hyperandrogenism and no gynecological or menstrual abnormalities ${ }^{[4]}$ were all ruled out. In case 3 the patient was diagnosed with idiopathic hirsutism without underlying hyperandrogenism. The patient had not been using any medication nor did she use any cosmetic cream containing hormones. As idiopathic hirsutism may be caused by increased $5 \alpha$-reductase activity in the pilosebaceous unit, ${ }^{[5]}$ and thus 'increased sensitivity' to androgens, hirsutism can exist in the absence of other signs of virilization (as found in our patient). The patients in cases 2 and 4 were diagnosed with PCOS according to the requirements for 
diagnosis outlined in the Rotterdam European Society of Human Reproduction and Embriology/American Society for Reproductive Medicine PCOS consensus guidelines, ${ }^{[1]}$ with the diagnosis based on the following: clinical signs of hyperandrogenization (acne in both cases, mild hirsutism in case 4); biochemical signs of hyperandrogenization (direct evidence in case 2 of elevated androgen serum levels and supporting evidence in case 4 of elevated androstenedione levels and elevated LH levels and an LH:FSH ratio, which support evidence for the diagnosis); ${ }^{[1,3]}$ oligo or anovulation (amenorrhea in case 2 [a sign of anovulation] and irregular periods in case 4) and ultrasound imaging showing polycystic ovaries. The ferrotherapy administered to the patient in case 2 would not have interfered with the results of laboratory tests. In both patients with PCOS, androgensecreting neoplasms could be excluded because of the absence of frank virilization and lack of rapid onset of hyperandrogenism. ${ }^{[3]}$ Neither patient had acanthosis nigricans, a cutaneous sign of hyperinsulinemia and thus were not suspected to have severe insulin resistance, which may co-occur with hyperandrogenism. ${ }^{[3]}$ Finally, rare possible causes of excess androgen, for example congenital adrenal hyperplasia, androgen-secreting tumors and Cushing's syndrome were not suspected based on clinical and/ or laboratory examinations and were ruled out in cases 1, 3 and 4 .

Among the pharmacological treatments available to manage or treat the dermatological disorders in the patients in our case series, a rational treatment choice would be hormonal therapies that target androgen excess (in which androgen excess is found, e.g. in acne $)^{[9]}$ or those that target pilosebaceous unit sensitivity to androgens (in which increased $5 \alpha$-reductase activity is found, e.g. in idiopathic hirsutism). ${ }^{[14]}$

Oral hormonal therapies investigated for female androgenetic alopecia have had varied success. The $5 \alpha$-reductase inhibitor (a testosterone conversion blocker) finasteride does not appear to be effective, at least in postmenopausal women (at a dose of $1 \mathrm{mg}){ }^{[15-17]}$ The oral anti-androgens (androgen receptor antagonists) spironolactone ${ }^{[18]}$ and cyproterone acetate (alone or when given in combination with an estrogen ${ }^{[18-20]}$ have been shown to be effective in limiting the progression of hair loss or improve hair growth, and have been used off-label for many years despite a lack of evidence from well-designed studies. The use of anti-androgens requires the use of good contraception because of the risk of feminization of a fetus. ${ }^{[21]}$ Evidence of their use from well-designed studies is lacking. Limited evidence suggests hormonal therapy is helpful even in patients with FPB with normal hormone levels. ${ }^{[2]}$ Currently, the only approved agent for female androgenetic alopecia is a non-hormonal therapy (minoxidil). ${ }^{[2]}$ None of the pharmacological treatments for FPB result in full hair recovery, and they are therefore used indefinitely to prolong their effect. ${ }^{[22]}$ Similarly, the management of hirsutism requires ongoing treatment with cosmetic (e.g. topical eflornithine hydrochloride) and physical measures (such as electrolysis, laser hair removal) and/or hormonal therapy ${ }^{[8]}$ Anti-androgens (e.g. spironolactone, cyproterone acetate) have been investigated in this indication but not in randomized controlled trials, and again, their use is limited by the potential for feminization of male fetuses. ${ }^{[8,21]}$ Combined oral contraceptives (COCs; combination of an estrogen and a progestin) are the first line of pharmacological treatment of hirsutism, particularly in young women who do not want to become pregnant. ${ }^{[21]} \mathrm{COCs}$ that may be used include those with low androgenic activity, such as ethinylestradiol in combination either with drospirenone (Yasmin), norgestimate (OrthoCyclen) or ethynodiol diacetate (Demulen 1-50).${ }^{[8]}$ Cyproterone has also been combined with ethinylestradiol in a contraceptive (Dianette) and is approved for use in moderately severe hirsutism, but is associated with body weight gain, reduced libido and breast tenderness. ${ }^{[23]}$

Two patients (cases 2 and 4) had PCOS and sought treatment for their acne (one of these patients also wanted birth control); treatment for associated fertility problems/anovulation was not requested.

Acne may be treated with topical and/or systemic drugs. Topical agents include retinoids, antibiotics or benzoyl peroxide. Systemic agents include antibiotics or retinoids, alone or in combination with COCs. COCs are considered firstline treatment in women with acne who desire contraception provided no contraindications exist; ${ }^{[24]}$ compared with placebo, COCs reduce acne lesion counts, lesion severity and self-assessed acne. ${ }^{[25]}$ COCs containing a progestin with antiandrogenic effects or an anti-androgen (e.g. cyproterone, drospirenone and chlormadinone) should be considered in the first place. ${ }^{[26]}$ Numerous studies support the use of COCs composed of low-dose estrogens (defined as ethinylestradiol $<50 \mu \mathrm{g}$ ) plus anti-androgenic agents or progestins with anti-androgenic

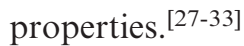

$\mathrm{EE} / \mathrm{CMA}$ is composed of a low-dose estrogen component (ethinylestradiol) and an anti-androgenic component (chlormadinone acetate), and was selected as an appropriate hormone therapy for each of the patients in this case series because, like other COCs, the estrogen component reduces androgen levels via their action on other hormones ( $\mathrm{LH}, \mathrm{FSH}$, sex hormonebinding globulin), and anti-androgenic progestins have androgen receptor and/or $5 \alpha$-reductase inhibitory activity. ${ }^{[34]}$ $\mathrm{EE} / \mathrm{CMA}$ has not been investigated in clinical trials in patients with FPB or hirsutism, but subgroups of patients receiving $\mathrm{EE} / \mathrm{CMA}$ in trials evaluating its efficacy in the treatment of 
acne and/or seborrhea have reported beneficial effects (as described in detail in the accompanying review article. ${ }^{[29,35,36]}$ All four patients were successfully treated with EE/CMA. It was also well tolerated; no treatment-related adverse events were reported by any of the patients in this case series.

EE/CMA has a favorable hormone profile, and appears to improve acne and other dermatological conditions; it is also well tolerated and patient compliance is generally high. ${ }^{[27,29,31,33]}$ Although confirmatory evidence of its efficacy in hirsutism and androgenetic alopecia in large, well-controlled studies is required, EE/CMA may be a valuable option among available COCs in the management of hirsutism, androgenetic alopecia and acne.

\section{Acknowledgments}

Medical writing assistance was provided by Tracy Harrison of inScience Communications, a Wolters Kluwer business. This assistance was funded by Faes Farma. All authors contributed to and approved the Introduction and Conclusions. The authors have no conflicts of interest that are directly relevant to the contents of this paper.

\section{References}

1. Azziz R, Carmina E, Dewailly D, et al. The Androgen Excess and PCOS Society criteria for the polycystic ovary syndrome: the complete task force report. Fertil Steril 2009 Feb; 91 (2): 456-88

2. Scheinfeld N. A review of hormonal therapy for female pattern (androgenic) alopecia [Commentary]. Dermatol Online J 2008; 14 (3): 1

3. Price VH. Androgenetic alopecia in women. J Investig Dermatol Symp Proc 2003 Jun; 8 (1): 24-7

4. Rivera R, Guerra-Tapia A. Manejo de la alopecia androgenetica en mujeres pomenopausicas. Actas Dermosifiliogr 2009; 99: 257-61

5. Blume-Peytavi U, Blumeyer A, Tosti A, et al. S1 guideline for diagnostic evaluation in androgenetic alopecia in men, women and adolescents. $\mathrm{Br}$ J Dermatol 2011 Jan; 164 (1): 5-15

6. Futterweit W, Dunaif A, Yeh HC, et al. The prevalence of hyperandrogenism in 109 consecutive female patients with diffuse alopecia. Journal of the American Academy of Dermatology 1988 Nov; 19 (5 Pt 1): 831-6

7. Olsen EA. Female pattern hair loss. Journal of the American Academy of Dermatology 2001; 45 (3 (Suppl 1)): S70-80

8. Rosenfield RL. Clinical practice. Hirsutism. N Engl J Med 2005 Dec 15; 353 (24): $2578-88$

9. Archer JS, Chang RJ. Hirsutism and acne in polycystic ovary syndrome. Best Pract Res Clin Obstet Gynaecol 2004 Oct; 18 (5): 737-54

10. Wendelin DS, Pope DN, Mallory SB. Hypertrichosis. Journal of the American Academy of Dermatology 2003 Feb; 48 (2): 161-79; quiz 80-1

11. Revised 2003 consensus on diagnostic criteria and long-term health risks related to polycystic ovary syndrome. "Rotterdam ESHRE/ASRM-Sponsored PCOS Consensus Workshop Group”. Fertil Steril 2004 Jan; 81 (1): $19-25$

12. Oberemok SS, Shalita AR. Acne vulgaris, I: pathogenesis and diagnosis. Cutis 2002 Aug; 70 (2): 101-5

13. Grünenthal GmbH. Belara ${ }^{\circledR}$ : highlights of the product's properties [product monograph]. Aachen: Grünenthal GmbH, 2001

14. Azziz R, Carmina E, Sawaya ME. Idiopathic hirsutism. Endocr Rev 2000 Aug; 21 (4): $347-62$
15. Price VH, Roberts JL, Hordinsky M, et al. Lack of efficacy of finasteride in postmenopausal women with androgenetic alopecia. Journal of the American Academy of Dermatology 2000 Nov; 43 (5 Pt 1): 768-76

16. Whiting DA, Waldstreicher J, Sanchez M, et al. Measuring reversal of hair miniaturization in androgenetic alopecia by follicular counts in horizontal sections of serial scalp biopsies: results of finasteride $1 \mathrm{mg}$ treatment of men and postmenopausal women. J Investig Dermatol Symp Proc 1999 Dec; 4 (3): 282-4

17. Thai KE, Sinclair RD. Finasteride for female androgenetic alopecia. Br J Dermatol 2002 Oct; 147 (4): 812-13

18. Sinclair R, Wewerinke M, Jolley D. Treatment of female pattern hair loss with oral antiandrogens. Br J Dermatol 2005 Mar; 152 (3): 466-73

19. Peereboom-Wynia JD, van der Willigen AH, van Joost T, et al. The effect of cyproterone acetate on hair roots and hair shaft diameter in androgenetic alopecia in females. Acta Derm Venereol 1989; 69 (5): 395-8

20. Vexiau P, Chaspoux C, Boudou P, et al. Effects of minoxidil 2\% vs. cyproterone acetate treatment on female androgenetic alopecia: a controlled, 12-month randomized trial. Br J Dermatol 2002 Jun; 146 (6): 992-9

21. Griffing G. Hirsutism Treatment \& Management. 2010 [cited 20119 May]; Available from: http://emedicine.medscape.com/article/121038-treatment

22. DeVillez RL, Jacobs JP, Szpunar CA, et al. Androgenetic alopecia in the female. Treatment with 2\% topical minoxidil solution. Arch Dermatol 1994 Mar; 130 (3): 303-7

23. Bayer plc. Dianette summary of product characteristics. 2010 [cited 201111 May]; Available from: http://www.medicines.org.uk/EMC/medicine/1814/ SPC/Dianette

24. Berson DS, Chalker DK, Harper JC, et al. Current concepts in the treatment of acne: report from a clinical roundtable. Cutis 2003 Jul; 72 (1 Suppl): 5-13

25. Arowojolu AO, Gallo MF, Lopez LM, et al. Combined oral contraceptive pills for treatment of acne. Cochrane Database Syst Rev 2009; (3): CD004425

26. Homburg R. Polycystic ovary syndrome. Best Pract Res Clin Obstet Gynaecol 2008 Apr; 22 (2): 261-74

27. Brucker C, Hedon B, The HS, et al. Long-term efficacy and safety of a monophasic combined oral contraceptive containing $0.02 \mathrm{mg}$ ethinylestradiol and $2 \mathrm{mg}$ chlormadinone acetate administered in a 24/4-day regimen. Contraception 2010 Jun; 81 (6): 501-9

28. Koltun W, Lucky AW, Thiboutot D, et al. Efficacy and safety of $3 \mathrm{mg}$ drospirenone $/ 20 \mathrm{mcg}$ ethinylestradiol oral contraceptive administered in 24/4 regimen in the treatment of acne vulgaris: a randomized, double-blind, placebo-controlled trial. Contraception 2008 Apr; 77 (4): 249-56

29. Plewig G, Cunliffe WJ, Binder N, et al. Efficacy of an oral contraceptive containing EE $0.03 \mathrm{mg}$ and CMA $2 \mathrm{mg}$ (Belara) in moderate acne resolution: a randomized, double-blind, placebo-controlled phase III trial. Contraception 2009 Jul; 80 (1): 25-33

30. Pushparajah DS, Rohm P, Hoschen K, et al. Safety data and beneficial effects of the combined oral contraceptive ethinylestradiol $0.03 \mathrm{mg} /$ chlormadinone acetate $2 \mathrm{mg}(\operatorname{Belara}(\mathrm{R}))$ : a 13-cycle, observational study in routine clinical practice. Clin Drug Investig 2011; 31 (2): 121-34

31. Sabatini R, Orsini G, Cagiano R, et al. Noncontraceptive benefits of two combined oral contraceptives with antiandrogenic properties among adolescents. Contraception 2007 Nov; 76 (5): 342-7

32. Uras R, Orru M, Pani F, et al. Endocrinological, metabolic and clinical features of treatment with oral contraceptive formulation containing ethinylestradiol plus chlormadinone acetate in nonobese women with polycystic ovary syndrome. Contraception 2010 Aug; 82 (2): 131-8

33. Zahradnik HP, Hanjalic-Beck A. Efficacy, safety and sustainability of treatment continuation and results of an oral contraceptive containing $30 \mathrm{mcg}$ ethinyl estradiol and $2 \mathrm{mg}$ chlormadinone acetate, in long-term usage (up to 45 cycles) - an open-label, prospective, noncontrolled, office-based phase III study. Contraception 2008 May; 77 (5): 337-43

34. Shaw JC. Acne: effect of hormones on pathogenesis and management. Am J Clin Dermatol 2002; 3 (8): 571-8 
35. Worret I, Arp W, Zahradnik HP, et al. Acne resolution rates: results of a singleblind, randomized, controlled, parallel phase III trial with EE/CMA (Belara) and EE/LNG (Microgynon). Dermatology 2001; 203 (1): 38-44

36. Guerra-Tapia A, Sancho B. Ethinylestradio/Chlormadione Acetate: dermatological benefits. Am J Clin Dermatol 2011; 12 (Suppl. 1): 3-11

Correspondence (Case Report 1): Dr Mercedes Gómez Vázquez, Cami del Cementiri n12 Sant Vicenç de Montalt, 08394 Barcelona, Department of Dermatology Hospital Municipal of Badalona, Spain.

Email: mercedesgomezvazquez@gmail.com
Correspondence (Case Report 2): Dr Marta Lamarca, Department of Gynecology, Miguel Servet University Hospital, Zaragoza, Spain.

Email: m.lamarca@yahoo.es

Correspondence (Case Report 3): Dr Sebastián Romero Ruiz, Hospital of Móstoles, Department of Obstetrics and Gynecology C/Rio Júcar S/N, 28935 Móstoles, Madrid, Spain.

Email: seromero98@yahoo.com

Correspondence (Case Report 4): Dr Eduardo Vilar-Checa, C/ Pintor Cabrera, $175^{\mathrm{a}} \mathrm{A}, 03003$ Alicante, Spain.

Email: vilar.checa@umh.es 